\title{
Strain-specific modification of lethality in fucose-deficient mice
}

\author{
Daniel J. Becker, ${ }^{1}$ Jay T. Myers, ${ }^{2}$ Melissa M. Ruff, ${ }^{3}$ Peter L. Smith, ${ }^{2,3}$ Brenda \\ W. Gillespie, ${ }^{4}$ David Ginsburg, ${ }^{1,2,5,6}$ and John B. Lowe ${ }^{1,2,3}$
}

\begin{abstract}
${ }^{1}$ Graduate Program in Cellular and Molecular Biology, University of Michigan Medical School, Ann Arbor, Michigan 48109, USA
${ }^{2}$ Howard Hughes Institute, University of Michigan Medical School, Ann Arbor, Michigan 48109, USA

${ }^{3}$ Department of Pathology, University of Michigan Medical School, Ann Arbor, Michigan 48109, USA

${ }^{4}$ Department of Biostatistics, University of Michigan Medical School, Ann Arbor, Michigan 48109, USA

${ }^{5}$ Department of Human Genetics, University of Michigan Medical School, Ann Arbor, Michigan 48109, USA

${ }^{6}$ Department of Internal Medicine, University of Michigan Medical School, Ann Arbor, Michigan 48109, USA
\end{abstract}

Received: 28 June 2002 / Accepted: 15 October 2002

\section{Abstract}

The FX locus encodes an essential enzyme in the de novo pathway of GDP-fucose biosynthesis. Mice homozygous for a targeted mutation of the $F X$ gene manifest a host of pleiotropic abnormalities including a lethal phenotype that is almost completely penetrant in heterozygous intercrosses on a mixed genetic background. Here we have investigated genetic suppression of $F X$-mediated lethality. Reduced recovery of heterozygous mice was observed while backcrossing the null $F X$ allele to $\mathrm{C} 57 \mathrm{BL} / 6 \mathrm{~J}(\mathrm{~B} 6)$, but was less dramatic in an outcross to CASA/Rk and absent in an outcross to $129 \mathrm{~S} 1 /$ SvImJ, indicating that genetic background modifies survival of $\mathrm{FX}+/-$ progeny. Substantial strain-specific differences in pre- and postnatal survival of FX-/- progeny were also detected in heterozygous crosses of C57BL/6J congenic, 129S1B6F1, and B6CASAF1 mice. Specifically, intrauterine survival of $\mathrm{FX}-/-$ mice was greatly increased during a heterozygous intercross on a uniform C57BL/6J genetic background compared with survival on a hybrid genetic background consisting of a mixture of C57BL/6J and 129S2/SvPas. In addition, statistically significant clustering of $\mathrm{FX}-/-$ progeny into litters and specific breeding cages was noted during a B6CASAF1 FX+/- intercross, suggesting a rare mechanism for modifier gene action in which parentally expressed genes define the phenotype, in this case the survival potential, of mutant offspring. Our results disclose that lethality in $F X$ mutant mice is determined by one or more strainspecific modifier loci.

Correspondence to: J.B. Lowe at Howard Hughes Medical Institute, 3510 MSRB I, 1150 W. Medical Center Drive, Ann Arbor, MI 48109-0650, USA. E-mail: johnlowe@umich.edu

\section{Introduction}

Precise correlation of genotype with phenotype is often difficult owing to factors such as allelic variation, locus heterogeneity, genetic background, and environmental effects. This has led some to propose that nearly all monogenic disorders are actually complex traits (Dipple and McCabe 2000b; Scriver and Waters 1999). In mice with mutations at specific loci, numerous examples of genetic background effects have been described in which genes from a particular inbred strain modify the expression of mutant phenotypes. Despite a recent focus on the importance of modifier genes as determinants of phenotypic variability in mammals, only a handful of modifier loci have been identified in either humans or mice (Dipple and McCabe 2000a; Nadeau 2001; Pearson 2002).

The $F X$ protein is a ubiquitously expressed epimerase-reductase enzyme necessary for the de novo biosynthesis of GDP-fucose from GDP-mannose (Tonetti et al. 1996). We have previously described mice with a targeted disruption of the $F X$ locus that are unable to convert GDP-mannose to GDP-fucose and display a corresponding absence of fucose from cellular glycoconjugates (Smith et al. 2002). FX-/- mice exhibit a number of abnormalities including growth retardation, infertility, neutrophilia with altered myelopoiesis, diarrhea with histological correlates resembling inflammatory bowel disease, and partially penetrant embryonic lethality. With the exception of the embryonic lethal phenotype, all of the FX-/- phenotypes recognized thus far are reversed by dietary fucose supplementation, which restores synthesis of GDP-fucose through a normally quiescent salvage pathway and thus corrects the fucosylated glycan deficiency. 
Intercross of $\mathrm{FX}+/-$ mice of mixed genetic background (C57BL/6J and 12982/SvPas strains) yields a substantial reduction in live $\mathrm{FX}-/-$ progeny compared with the value expected by Mendelian inheritance, with only $5-7 \%$ present at late embryonic stages and only $2 \%$ recovered at weaning (postnatal day 21; Smith et al. 2002). Thus, lethality occurs both in utero and during the postnatal nursing period. Dietary administration of exogenous fucose to $\mathrm{FX}+/$ - parental mice during gestation and subsequent nursing of pups do not alter the frequency of $\mathrm{FX}-/-$ progeny at weaning. Rare $\mathrm{FX}-/-$ mice survive to adulthood and retain their fertility when maintained on a fucose-supplemented diet. Surprisingly, when two such FX-/- male mice were mated with $\mathrm{FX}+/-$ females, a significantly higher proportion of $\mathrm{FX}-/-$ progeny $(27-33 \%)$ were recovered at weaning, regardless of whether the breeding cages were supplied with exogenous fucose. Further, intercross of FX-/- mice yielded litters of FX-/progeny that were similar in size to those obtained from wild-type mice of comparable genetic background (Smith et al. 2002). These results suggested the existence of one or more major modifier loci responsible for rescuing $\mathrm{FX}-/-$ mice from lethality.

In this study, we explore the basis for the variable penetrance of lethality in FX-/- mice. Congenic mice harboring the null $F X$ allele were created on the C57BL/6J background and outcrossed to 129S1/ SvImJ and CASA/Rk mice. These breeding experiments revealed complex strain-specific effects on survival of $\mathrm{FX}+/-$ and $\mathrm{FX}-/-$ mice. Taken together, our results demonstrate that lethality of $\mathrm{FX}+/-$ and $\mathrm{FX}-/-$ mice is controlled by one or more strainspecific modifier gene(s).

\section{Materials and methods}

Mice. All mice were bred in a specific pathogen-free facility at the University of Michigan Medical School. C57BL/6J, CASA/Rk, and 12981/SvImJ (previously named 129S3/SvImJ; Jackson stock \#002448) mice were purchased from The Jackson Laboratory (Bar Harbor, Me.). Creation of a null allele

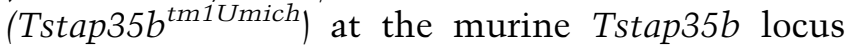
has been previously described (Smith et al. 2002). For clarity, the common name for this locus, $F X$, is used throughout this study. The B6.129 congenic FX+/mice used in the crosses depicted in Tables 2 and 4 had been crossed to C57BL/6J for nine generations (N9) and are, therefore, more precisely known as incipient congenic. Fucose supplementation (Tables 2 and 3) was done with chow containing $2.5 \%$ (wt/ wt) fucose as described (Smith et al. 2002). For neonatal genotyping, breeding cages were checked for newborn pups each morning at 7AM and periodically throughout the day. Newborns, including dead pups, were removed and sacrificed, and tissue from the lower body was collected for preparation of genomic DNA. When multiple, partially cannibalized pups were present in a single cage, tissue was collected only when it could be assigned unambiguously to individual pups. Genotype at the $F X$ locus was determined by PCR as described (Smith et al. 2002). Statistical analysis of breeding data was done with SAS software at the Center for Statistical Consultation and Research at the University of Michigan.

Genome scans. Genomic DNA was prepared from tail and tissue samples as described (Strauss 1998). A DNA pooling strategy was used as described (Taylor et al. 1994). Pools representing 37 mice were created by mixing equivalent amounts of DNA (2-5 $\mu \mathrm{g})$, and $100 \mathrm{ng}$ of each pool was used for PCR. Markers were selected from the Whitehead Institute/Massachusetts Institute of Technology (http:// www-genome.wi.mit.edu/cgi-bin/mouse/index) and Center for Inherited Disease Research (http:// www.cidr.jhmi.edu/mouse/mouse.html) databases. MIT primer pairs were purchased from Research Genetics. Marker map position and order was determined with the Jackson Laboratory Mouse Genome Database (http://www.informatics.jax.org//. Primers were labeled with $\left[\gamma_{-}{ }^{32} \mathrm{P}\right]-\mathrm{ATP}(\mathrm{NEN})$ and T4 polynucleotide kinase (New England Biolabs). Simple sequence repeats were amplified in $10-\mu \mathrm{L}$ reactions with Platinum ${ }^{\circledR}$ Taq DNA polymerase /Gibco BRL) and standard reaction conditions. Radiolabeled products were resolved on $8 \%$ polyacrylamide/urea sequencing gels. In some cases, unlabeled primers were used in $25-\mu \mathrm{L}$ reactions, and products were resolved on 3\% Metaphor agarose (BioWhittaker Molecular Applications)/1\% standard agarose (Gibco BRL) gels. Additional markers were analyzed with fluorescently labeled primers and were resolved with an ABI model 3700 DNA sequencer in the University of Michigan DNA Sequencing Core.

\section{Results}

Heterozygous and homozygous lethality of mutant mice on C57BL/6J background. Our initial characterization of mice with a targeted deletion at the $F X$ locus suggested that a major modifier gene was altering the penetrance of the lethal phenotype in FX-/- mice (Smith et al. 2002). These FX+/- and FX-/- mice were on a hybrid genetic background consisting of $60-80 \%$ C57BL/6J and 20-40\% 129S2/ SvPas, the strain from which the D3 embryonic stem cells used in gene targeting were derived (Simpson 
Table 1. Progeny of FX+/- backcross to $\mathrm{C} 57 \mathrm{~B} / 6 \mathrm{~J}$. No fucose supplementation; genotyping at weaning

\begin{tabular}{llrrr}
\hline & + /- parent & \multicolumn{1}{c}{ n } & WT & Het \\
\hline N2 & M & 9 & $8(89 \%)$ & $1(11 \%)$ \\
N2 & F & 36 & $19(53 \%)$ & $17(47 \%)$ \\
N3 & M & 72 & $53(74 \%)$ & $19(26 \%)$ \\
N4 & M & 131 & $80(61 \%)$ & $51(39 \%)$ \\
N5 & M & 93 & $68(73 \%)$ & $25(27 \%)$ \\
N6 & M & 141 & $78(55 \%)$ & $63(45 \%)$ \\
N7 & F & 108 & $63(58 \%)$ & $45(42 \%)$ \\
N8 & M & 168 & $102(61 \%)$ & $66(39 \%)$ \\
N9 & 124 & $85(69 \%)$ & $39(31 \%)$ \\
\hline Total & & 882 & $556(63 \%)$ & $326(37 \%)$ \\
\hline
\end{tabular}

The sex $(\mathrm{M}=$ male, $\mathrm{F}=$ female $)$ of the $\mathrm{FX}+/-(\mathrm{Het})$ mice used from the previous generation for mating to wild-type (WT) C57BL/6J mice is shown for each generation (N). The number of progeny at each generation is indicated (n). Genotypes of progeny mice were determined at weaning (postnatal day 21 ). The binomial probability ( $z$ value) was calculated based on the Mendelian frequency $(50 \%$ WT, $50 \%$ Het) and used to calculate the one-tailed P-value. For the N2-N9 total, $P$-value $<10^{-3}$. Het; heterozygote. WT; wild-type

Table 2. Progeny of N9 B6.129 congenic FX+/- $\times$ FX+/- intercross. Fucose-supplemented chow; genotyping at weaning

\begin{tabular}{lccccl}
\hline & Male & Female & Total & \% Observed & \% Expected \\
\hline$+/+$ & 78 & 82 & 160 & 45.7 & 25 \\
$+/-$ & 105 & 83 & 188 & 53.7 & 50 \\
$-/-$ & 1 & 1 & 2 & 0.6 & 25 \\
\hline $\mathrm{n}$ & 184 & 166 & 350 & & \\
\hline
\end{tabular}

Chi-square analysis was used to compare the observed values with the values predicted by Mendelian inheritance patterns. $P$-value $<10^{-3}$.

et al. 1997). To determine whether specific modifier gene(s) from either the C57BL/6J or 129S2/SvPas genetic backgrounds were conferring a survival advantage to FX-/- progeny, we performed a genome scan in an effort to locate regions of either genome that were preferentially inherited by FX-/- survivors. Pooled DNA samples representing 37 FX-/progeny of $\mathrm{FX}-/-\times \mathrm{FX}+/-$ crosses were compared with pooled samples of $37 \mathrm{FX}+/$ - littermates by using 42 simple sequence length polymorphism (SSLP) markers distributed throughout the 19 mouse autosomes. Evidence for linkage of specific chromosomal regions to survival of FX-/- mice was not uncovered. This analysis, however, was complicated by the fact that all of the hybrid background FX-/- progeny were derived from two male FX-/- founders.

To obtain the targeted $F X$ allele on a uniform genetic background, the original chimeric mice produced from the targeted embryonic stem cell clone were crossed to C57BL/6J mice for nine generations to create N9 B6.129 congenic $\mathrm{FX}+/-$ mice. During the backcross, we observed a significant reduction in the number of $\mathrm{FX}+/-$ mice recovered at weaning relative to $\mathrm{FX}+/+$ animals $137 \%$ heterozygotes for N2-N9 combined vs. 50\% expected, $\mathrm{n}=882$, $p<0.001$ ) (Table 1). FX+/- mice thus exhibit a partially penetrant lethal phenotype in addition to $\mathrm{FX}-/-$ mice. The ratio of $\mathrm{FX}+/+$ to $\mathrm{FX}+/-$ progeny varied from generation to generation, approaching
$1: 1$ in some generations while exceeding $2: 1$ in others, with no consistent trend as generation number increased. The variation in heterozygous survival in different generations could be related to the fact that only a relatively small cohort of $\mathrm{FX}+/$ - progeny was selected at each generation for breeding to C57BL/6J. The loss of $\mathrm{FX}+/-$ mice during backcrossing is consistent with our earlier finding that the ratio of $\mathrm{FX}+$ /+ to $\mathrm{FX}+/-$ progeny from hybrid $\mathrm{FX}+/-\times \mathrm{FX}+/-$ crosses is variably altered from the expected $1: 2$ ratio (Smith et al. 2002).

We next intercrossed N9 B6.129 congenic FX+/mice to determine whether FX-/- mice could be recovered on a uniform $\mathrm{C} 57 \mathrm{BL} / 6 \mathrm{~J}$ background. As we had observed in $\mathrm{FX}+/-\times \mathrm{FX}+/-$ intercrosses with hybrid background mice, only a very low frequency $(0.6 \%)$ of FX-/- mice survived to weaning (Table 2). We also observed a substantial decrease in the survival of $\mathrm{FX}+/-$ mice with the ratio of wild-type to heterozygous progeny only 1:1.2 rather than the $1: 2$ ratio expected for a heterozygous intercross $(p<0.001)$. One of the two surviving FX $-/-$ mice was male and was subsequently mated with several N9 B6.129 congenic FX+/- females. Fourteen FX-/mice were recovered from 51 total progeny (Table 3), a finding similar to the results of the hybrid background $\mathrm{FX}-/-\times \mathrm{FX}+/-$ cross in which approximately one-third of total progeny were FX-/- (Smith et al., 2002). These data were surprising because back- 
Table 3. Progeny of N9 B6.129 congenic FX $-/-\times$ FX+/- cross. Fucose-supplemented chow; genotyping at weaning

\begin{tabular}{llllll}
\hline & Male & Female & Total & \% Observed & \% Expected \\
\hline$+/-$ & 20 & 17 & 37 & 72.5 & 50 \\
$-/-$ & 9 & 5 & 14 & 27.4 & 50 \\
\hline $\mathrm{n}$ & 29 & 22 & 51 & & \\
\hline
\end{tabular}

The single male FX-/- mouse generated from the N9 B6.129 congenic FX+/- $\times$ FX+/- inter-cross (see Table 2) was mated with multiple $\mathrm{FX}+/-$ females. The binomial probability was calculated based on the expected Mendelian frequency $(50 \% \mathrm{FX}+/-, 50 \% \mathrm{FX}-/-)$ and used to calculate the one-tailed $P$-value. $P$-value $<10^{-3}$.

Table 4. Progeny of N9 B6.129 congenic FX+/- outcrosses. No fucose supplementation; genotyping at weaning

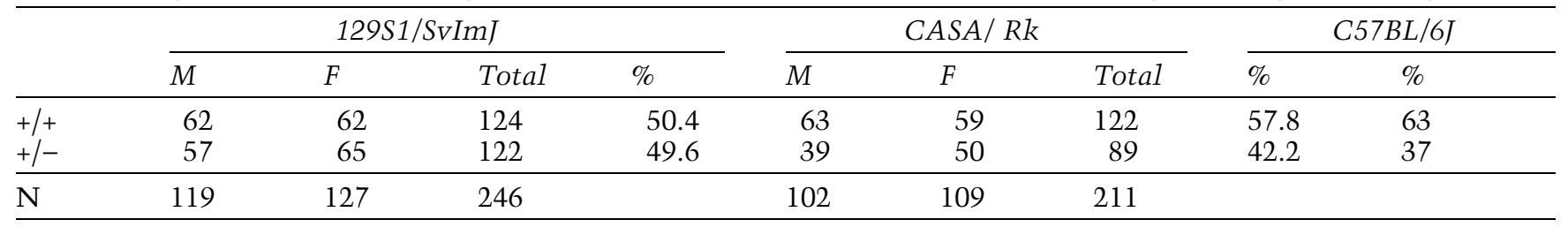

Male congenics were used for the cross to $129 \mathrm{~S} 1 / \mathrm{SvImJ}$. Female congenics were used for the cross to CASA/Rk. For comparison, the genotypic data from the backcross to C57BL/6J is shown on the right (combined N2-N9; see Table 1). The binomial probability was calculated based on the expected Mendelian frequency (50\% FX+/+, 50\% FX+/-). For the 129S1/SvImJ outcross, the one-tailed $P$-value $=0.474$. For the CASA/Rk outcross, the one-tailed $P$-value $=0.013$.

crossing to C57BL/6J did not substantially increase or decrease the frequency of weaned FX-/- survivors, as might be expected as the genetic background became more uniformly derived from C57BL/6J.

129S1/SvImJ and CASA/Rk genetic backgrounds partially rescue $F X$-mediated lethality. We sought to determine whether the 129S2/SvPas genome contains a major modifier gene for survival of FX-/- mice. During the backcross, a 129-derived congenic interval could have been retained surrounding not only the $F X$ locus but also surrounding a putative modifier locus. The 129-derived modifier locus would be required for survival of FX-/- mice and would also confer a survival advantage to $\mathrm{FX}+/-$ mice, potentially explaining the nearly $2: 1$ ratio of $\mathrm{FX}+/+$ to $\mathrm{FX}+/-$ mice during the backcross. To test this hypothesis, we outcrossed N9 B6.129 congenic $\mathrm{FX}+/-$ mice to $129 \mathrm{~S} 1 / \mathrm{SvImJ}$, a readily available strain that is closely related to $12982 /$ SvPas (Simpson et al. 1997). In agreement with the model outlined above, $\mathrm{FX}+/-$ mice were fully represented at weaning $(49.6 \%, \mathrm{n}=246)$ (Table 4$)$, suggesting that the 129 genome contains a gene or genes capable of fully rescuing $\mathrm{FX}+/-$ mice.

We also outcrossed N9 B6.129 congenic FX+/mice to CASA/Rk. This inbred strain was derived from a divergent subspecies of mice, Mus musculas castaneus, and is, therefore, highly informative for mapping experiments. As shown in Table 4, we observed a greater recovery of $\mathrm{FX}+/-$ mice $142.2 \%$, $\mathrm{n}=211$ ) in the CASA/Rk outcross compared with the recovery from the backcross to C57BL/6J, but less complete than the recovery from the outcross to 129S1/SvImJ.

To determine whether loci from either the 129S1/SvImJ or CASA/Rk background increase the survival of $\mathrm{FX}-/-$ progeny, we intercrossed $\mathrm{FX}+/-\mathrm{F}_{1}$ mice. To our surprise, in light of the complete recovery of heterozygotes from the 129S1/SvImJ outcross, no FX-/- progeny were observed at weaning in the 129S1B6F1 FX+/- intercross ( $\mathrm{n}=112$ ) (Table 5). However, in agreement with the 129 S1/SvImJ outcross, the ratio of $\mathrm{FX}+/+$ to $\mathrm{FX}+/$ - progeny from the 129S1B6F1 intercross closely paralleled the expected $1: 2$ ratio. In the $\mathrm{B} 6 \mathrm{CASAF} 1 \mathrm{FX}+/-$ intercross, the frequency of $\mathrm{FX}-/-$ progeny was $4.8 \%$ at weaning $(\mathrm{n}=397)$ (Table 5). This represents a threefold increase over the recovery of $\mathrm{FX}-/-$ mice from hybrid $\mathrm{FX}+/-$ intercrosses and an eightfold increase over the recovery from the $\mathrm{B} 6.129$ congenic $\mathrm{FX}+/$ - intercross. Thus, survival of FX-/- mice to weaning is dramatically affected by genetic background.

Substantial perinatal death and cannibalization of pups was observed during the B6CASAF1 intercross. As we were concerned that postnatal death of $\mathrm{FX}-/-$ mice was reducing recovery of $\mathrm{FX}-/-$ mice, we began collecting tissue from all newborn mice, including dead pups, as soon after birth as possible. When the genotypes were determined during the neonatal period, the proportion of $\mathrm{FX}-/-$ animals recovered from the B6CASAF1 intercross increased to $8.7 \%$ (vs. $4.8 \%$ at weaning; $\mathrm{n}=206$ ) (Table 5), confirming that postnatal death partially contributes to the decreased prevalence of $\mathrm{FX}-/-$ mice at weaning. This $8.7 \%$ frequency of $\mathrm{FX}-/-$ progeny 
Table 5. Progeny of intercrosses between F1 FX+/- mice. No fucose supplementation

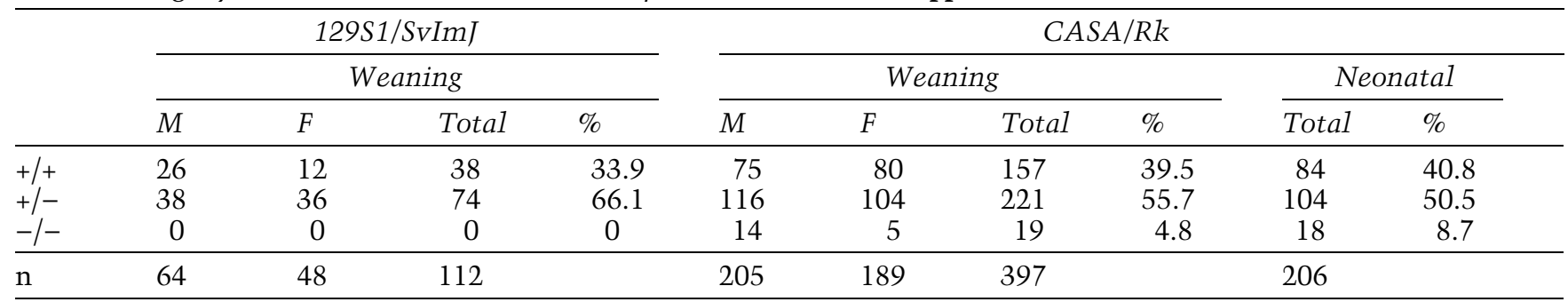

Genotypes were determined at weaning (postnatal day 21) or during the neonatal period (postnatal day 0.5). Chi-square analysis was used to compare the observed values with the values predicted by Mendelian inheritance patterns. $P$-value $<10^{-3}$ for all three crosses.

Table 6. Distribution of null progeny in litters from B6CASAF1 FX+/- intercross

\begin{tabular}{|c|c|c|c|c|c|c|c|}
\hline \multirow[b]{3}{*}{$\begin{array}{l}\text { No. of Nulls } \\
\text { per litter }\end{array}$} & \multicolumn{3}{|c|}{ Weaning } & & \multicolumn{3}{|c|}{ Neonatal } \\
\hline & \multicolumn{3}{|c|}{ (Null freq $=0.048)$} & & \multicolumn{3}{|c|}{$($ Null freq $=0.087)$} \\
\hline & $\begin{array}{l}\text { Expected } \\
\text { No. of litters }\end{array}$ & & $\begin{array}{l}\text { Observed } \\
\text { No. of litters }\end{array}$ & & $\begin{array}{l}\text { Expected } \\
\text { No. of litters }\end{array}$ & & $\begin{array}{l}\text { Observed } \\
\text { No. of litters }\end{array}$ \\
\hline 0 & 36.7 & $<$ & 41 & CLUSTERING & 14.7 & $<$ & 21 \\
\hline 1 & 12.6 & $>$ & 5 & REPULSION & 9.7 & $>$ & 1 \\
\hline 2 & 2.4 & $<$ & 4 & & 3.0 & $>$ & 2 \\
\hline 3 & 0.4 & $<$ & 2 & CLUUSTERING & 0.6 & $<$ & 4 \\
\hline$p$-value & & .00 & & & & .00 & \\
\hline
\end{tabular}

Litters were grouped based on whether the mice were genotyped at weaning (postnatal day 21) or during the neonatal period (postnatal day 0.5). Litters that contained only one or two pups were not included in this analysis. In a few cases, pups born to both mothers in a breeding triangle within a few days of each other could not be reliably assigned to distinct litters and were thus considered to be a single litter for this analysis. The combined frequency of null progeny for either weaning or neonatal genotyping was used to calculate the multinomial probability of each litter containing $0,1,2$, or 3 FX-/- progeny, based on the total number of pups in each individual litter. These values from each litter were summed to generate the expected number of litters containing $0,1,2$, or 3 FX-/- progeny and compared with the observed number of litters by using the chi-square test.

during the early neonatal period is reminiscent of our earlier finding from an intercross of hybrid $\mathrm{FX}+/-$ mice in which the frequency of $\mathrm{FX}-/-$ progeny was $5-7 \%$ at late embryonic stages (Smith et al. 2002).

To determine whether particular regions of the C57BL/6J or CASA/Rk genomes are correlated with survival of FX-/- mice, we examined pooled DNA samples from surviving B6CASAF2 FX-/- mice (from the cross depicted in Table 5; 19 weaned mice and 18 neonates), their $\mathrm{F}_{2} \mathrm{FX}+/+$ littermates, and equivalent $\mathrm{F}_{2} \mathrm{FX}+/+$ progeny from other litters with 65 SSLP markers distributed throughout the mouse genome. Each of the markers has distinct C57BL/6J, CASA/Rk, and 129S2/SvPas alleles. Except in the region of the FX locus on Chromosome (chr) 15, the proportion of C57BL/6J and CASA/Rk alleles was similar in all three pools and indistinguishable from the B6CASAF1 control (1:1 ratio). Additionally, we did not uncover evidence for a 129-derived modifier locus in the B6.129 congenic mice as $129 \mathrm{~S} 2 /$ SvPas alleles were detected only on Chr 15.

Non-random distribution of $\mathrm{FX}-/-$ mice into specific litters. During the B6CASAF1 FX+/- intercross, FX-/- progeny, although occurring infrequently overall, often were found with other FX-/mice in the same litter. Because this empirical observation appeared especially dramatic, we sought to determine whether the clustering of $\mathrm{FX}-/-$ progeny within litters was statistically significant. To determine the expected distribution of $\mathrm{FX}-/-$ progeny among the litters from the B6CASAF1 intercross, we calculated the probability that each litter would contain $0,1,2$, or $3 \mathrm{FX}-/$ - progeny based on the size of the litter and the overall frequency of $\mathrm{FX}-/-$ progeny. The expected number of litters of each class in the cross was obtained by summing these values. The observed distribution of FX-/- progeny among the litters was significantly different from the expected random distribution, both for litters geno- 
Table 7. Distribution of null progeny in breeding cages from B6CASAF1 FX+/- intercross

\begin{tabular}{lllll}
\hline Nulls per cage & \multicolumn{2}{l}{ Expected No. of cages } & & \multicolumn{2}{l}{ Observed No. of cages } \\
\hline 0 & 5.2 & $<$ & 15 & CLUSTERING \\
1 & 6.5 & $>$ & 0 & REPULSION \\
2 & 4.9 & $>$ & 0 & \\
3 & 2.9 & $>$ & 1 & \\
4 & 1.5 & $>$ & 1 & \\
5 & 0.6 & $<$ & 2 & CLUSTERING \\
6 & 0.2 & $<$ & 1 & \\
7 & 0.05 & $<$ & 2 & \\
$>7$ & 0.3 & - & 0 & \\
\hline
\end{tabular}

$p$-value

$<0.0001$

For each cage (breeding triangle), the multinomial probability for the cage to generate $0-7$ null progeny was calculated, based on the total number of pups born in each individual cage. The difference in the frequency of nulls based on the time at which the animals were genotyped (neonatal vs. weaning) was taken into account for each litter within the cage. These values from each cage were summed to generate the "Expected No. of cages" for each category of "Nulls per cage" and compared with the "Observed No. cages" by using the chisquare test.

typed at weaning and during the neonatal period (Table 6). These data suggested the possibility that certain B6CASAF1 FX+/- parents were better suited to giving rise to $\mathrm{FX}-/-$ progeny. Further supporting this notion, we also observed that particular breeding triangles (one male, two females) repeatedly generated FX-/- progeny, while other cages never generated an $\mathrm{FX}-/-$ mouse despite the fact that in many instances five or more litters and 30 or more pups had been genotyped. Strikingly, the same cages that had produced $\mathrm{FX}-/-$ mice while genotyping was performed at weaning continued to generate FX-/progeny during the neonatal genotyping phase. Thus, to determine whether FX-/- progeny were also clustered into particular breeding cages, we applied a statistical protocol similar to the method used for the litter analysis to calculate the expected distribution of $\mathrm{FX}-/-$ progeny among the 22 cages. As with our analysis of individual litters, there was a statistically significant difference between the observed distribution of $\mathrm{FX}-/$ - progeny among breeding cages and the expected distribution (Table 7). The ability to generate $\mathrm{FX}-/-$ progeny is thus strongly correlated with specific parent combinations, at least on a mixed C57BL/6:CASA background. This ability to generate $\mathrm{FX}-/$ - progeny could not be assigned to individual B6CASAF1 parents within a breeding cage, however, because the entire colony had been organized into breeding triangles, and the advancing age of the mice precluded extensive test crosses.

Increased intrauterine survival of $\mathrm{FX}-/-$ progeny during intercross of B6.129 congenic $\mathrm{FX}+$ /mice. Because we documented a substantial amount of postnatal death during the B6CASAF1 intercross, we repeated the intercross of heterozygous B6.129 congenic mice to determine the relative contribu- tions of intrauterine and postnatal demise to the low frequency of $\mathrm{FX}-/-$ progeny on the C57BL/6J background. When N12 B6.129 congenic FX+/- mice were intercrossed, the genotypic distribution of the $\mathrm{N} 12 \mathrm{~F} 1$ progeny at weaning was $63 \mathrm{FX}+/+(38.2 \%)$, $101 \mathrm{FX}+/-(61.2 \%)$, and $1 \mathrm{FX}-/-(0.6 \%)$, in accordance with the results of the N9 FX+/-intercross shown in Table 2. We then intercrossed 44 pairs of N12F1 $\mathrm{FX}+/-$ mice and genotyped the progeny during the neonatal period. Compared with the frequency at weaning, the overall frequency of N12F2 FX-/progeny was greatly increased, approaching the $25 \%$ value expected by a Mendelian inheritance pattern (22.3\%; $\mathrm{n}=471$; Table 8). Thus, on the C57BL/6J background, postnatal death is largely responsible for the reduced recovery of $\mathrm{FX}-/-$ mice at weaning.

\section{Discussion}

Targeted disruption of the $F X$ locus yields a conditional defect in GDP-fucose biosynthesis, impairment in expression of fucosylated glycans, and a partially penetrant lethal phenotype (Smith et al. 2002). Here, we have explored the genetic factors contributing to survival of $F X$ mutant mice. Our analyses demonstrate that genetic background substantially modifies the survival potential of $\mathrm{FX}+/-$ and $\mathrm{FX}-/-$ mice, both prenatally and postnatally.

While backcrossing the FX null allele to C57BL/ $6 \mathrm{~J}$, and in $\mathrm{FX}+/-$ intercrosses, we observed a significant loss of $\mathrm{FX}+/-$ progeny. Lethality of $\mathrm{FX}+/-$ mice was unexpected, as heterozygosity for a loss-offunction mutation in an enzyme-encoding gene, with retention of $50 \%$ of wild-type enzyme activity, is not typically deleterious to cellular or organismal function. The porphyrias, caused by haploinsufficiency of heme biosynthetic enzymes, are prominent exceptions to this paradigm. Although many of the 
Table 8. Progeny of N12F1 B6.129 congenic FX+/- $\times$ FX+/- intercross. No fucose supplementation, genotyping during neonatal period

\begin{tabular}{llll}
\hline & Progeny & \% Observed & \% Expected \\
\hline$+/+$ & 128 & 27.2 & 25 \\
$+/-$ & 238 & 50.5 & 50 \\
$-/-$ & 105 & 22.3 & 25 \\
$\mathrm{n}$ & 471 & & \\
\hline
\end{tabular}

Chi-square analysis was used to compare the observed values with the values predicted by Mendelian inheritance patterns. There is not a statistically significant difference between the observed distribution of genotypes and the expected distribution $(p=0.317)$.

phenotypes in porphyria can be attributed to toxic accumulation of intermediates in the heme biosynthetic pathway, some clinical findings in acute intermittent porphyria may be related to heme deficiency with consequent defects in heme-dependent biological processes (Kappas et al. 1995). Nucleotide sugar availability, including GDP-fucose synthesis, can be rate limiting for protein glycosylation (Eshel et al. 2000; Keppler et al. 1999; Marquardt et al. 1999; Toma et al. 1996; Smith et al. 2002). Thus, heterozygosity for a mutant $F X$ allele could lead to reductions in GDP-fucose biosynthesis, hypofucosylation of cellular proteins and lipids, and defects in pre- and postnatal development. When we outcrossed B6.129 congenic FX+/- mice to 129S1/ SvImJ, heterozygotes were completely represented in the $F_{1}$ progeny, providing evidence for one or more 129-derived modifier genes. However, no FX-/progeny were recovered at weaning from an intercross of 129S1B6F1 FX+/- mice, either because postnatal death confounded our ability to recover FX mutant mice on this genetic background or because independent genetic factors determine survival of $\mathrm{FX}+$ /- versus FX-/- mice.

Pronounced strain-dependent differences exist in the survival of $\mathrm{FX}-/-$ mice to weaning, (undetectable in the 129S1B6F1 intercross; $0.6 \%$ in the B6.129 congenics; $1.7-1.9 \%$ in the hybrid background; $4.8 \%$ in the B6CASAF1 intercross). These differences may be partially accounted for by differences in perinatal death (in the B6CASAF1 FX+/- intercross, recovery of $\mathrm{FX}-/$ - progeny was reduced from $8.7 \%$ in neonates to $4.8 \%$ at weaning, whereas in the B6.129 congenics, there was a $22.3 \%$ frequency of $\mathrm{FX}-/-$ neonates in the $\mathrm{N} 12 \mathrm{~F} 1 \mathrm{FX}+/-$ intercross compared to a $0.6 \%$ frequency of FX-/- weanlings in the N9 and N12 intercrosses). Variation in the ability of heterozygous mothers to supply FX mutant pups with fucose via milk may be an important determinant of rescue during the nursing period. FX-/- mice that do survive to weaning are runted and sickly, indicating that FX-/- pups may compete poorly with $\mathrm{FX}+/+$ and $\mathrm{FX}+/-$ littermates for access to teats and thus remain vulnerable to early demise. Other factors that may contribute to postnatal death include potentially strain-dependent maternal behaviors such as pup retrieval, nest building, and parental propensity for cannibalization, and subtle changes in the vivarium environment.

Parent-specific survival of FX-/- progeny in the B6CASAF1 FX+/- intercross is a peculiar phenomenon. Phenotypic variability among $F_{1}$ animals is difficult to rationalize because $F_{1}$ mice are genetically identical. However, the B6CASAF1 heterozygotes were not entirely homogeneous as a 129-derived congenic interval surrounds $F X$ and may encompass a modifier locus. Differences in postnatal maternal behavior do not appear to be solely responsible for the clustering of FX-/- progeny into particular litters or breeding cages, as the specificity of nullgenerating cages was retained when neonates were genotyped. Modifier genes expressed in the $\mathrm{FX}+/-$ mother may alter delivery of fucose to FX-/- embryos or otherwise impact survival in utero, thus invoking a rare mechanism for modifier effects whereby genes active in the mother directly determine the expression of a phenotype in her progeny. The genetic basis for this phenomenon could be due to modifier effects present in the C57BL/6J and 129 strain backgrounds, or could be an independent effect of genes contributed by the CASA/Rk and/or C57BL/6J genomes.

Our evidence indicates that strain-specific genetic modifiers are major determinants of the penetrance of FX-mediated lethality. First, substantial differences in heterozygous and homozygous lethality were observed in the outcross-intercross experiments on the $129 \mathrm{~S} 1 / \mathrm{SvImJ}$ and CASA/Rk genetic backgrounds. Second, clustering of B6CASAF2 FX-/- progeny into specific litters and breeding cages strongly suggests that one or more specific genetic loci, inherited from the B6.129 congenic $\mathrm{FX}+/-$ mice outcrossed to CASA/Rk, distinguished the B6CASAF1 FX+/- parents. Third, intrauterine survival of $\mathrm{FX}-/-$ progeny is greatly increased on the B6.129 congenic genetic background (Table 8) com- 
pared with the hybrid genetic background (Smith et al. 2002), demonstrating that survival of FX-/- mice improves after backcrossing to C57BL/6J. Despite compelling evidence that one or more loci account for variation in frequency of $\mathrm{FX}+/-$ and $\mathrm{FX}-/$ - progeny, our data do not yet identify a precise genetic mechanism. It is possible that a major recessive Mendelian modifier is responsible for survival of $F X$ mutant mice, but was not recognized in the genome scans performed on the hybrid background or B6CASAF2 progeny. However, the combined breeding results instead point toward multiple recessive modifiers, perhaps epistatically interacting, with weak but additive effects on $F X$-mediated lethality, or towards a small number of dominant modifier loci, which may not be detected in pooled genome scans.

Although survival of FX-/- progeny from a heterozygous cross of $\mathrm{B} 6.129$ congenic mice is nearly complete until the early postnatal period (Table 8 ), a substantial number of FX-/- mice on the hybrid background die prenatally, with only 5-7\% remaining at days 16.5-18.5 post-coitus (Smith et al. 2002). Death of FX-/- embryos may not be due to fucose deficiency, but rather to the absence of another, previously unrecognized function of the FX protein, or to the indirect metabolic consequences of FX protein deficiency. Indeed, spleens from FX-/- embryos (18.5 days post-coitus) gestated by heterozygous mothers express the fucose-containing ligands for E-selectin (Smith et al. 2002), demonstrating that FX-/- embryos acquire sufficient fucose, presumably from the maternal circulation via the placenta, to support expression of some fucosylated glycans. Nevertheless, the fact that normal-sized litters of FX-/- progeny are produced by fucose-fed FX-/mothers diminishes the likelihood that FX-/- embryonic lethality is independent of fucosylation. Instead, we propose that $F X$-mediated lethality is a direct consequence of incomplete restoration of fucosylated glycan expression. Efforts to confirm this possibility could include measurement of plasma fucose in such mothers, and in the fluids and cells of the embryos and placenta.

Restoration of fucosylation to FX mutant embryos is a complex process involving multiple interdependent events. Supply of fucose to FX-/embryos requires: i) production of free fucose and/or fucosylated glycoconjugates by the FX+/- mother; ii) secretion into maternal serum; iii) transplacental transfer; iv) dissemination throughout the developing embryo via the fetal circulation; v) transport into the cell via a specific plasma membrane fucose transporter or endocytosis of proteins; and, in the case of fucosylated glycoproteins, vi) release of fu- cose by lysosomal fucosidases. These processes during embryonic development are largely unexplored in mammals. Once fucose has been delivered to the cytosol of embryonic cells, it must be converted to GDP-fucose by enzymes of the salvage pathway, whose expression during embryogenesis is also unexplored. GDP-fucose synthesized in the cytosol is then transported into the Golgi lumen (Puglielli and Hirschberg 1999) to serve as a fucose donor in the synthesis of a wide variety of glycoconjugates. Fucose is a component of many glycolipids, is a terminal modification of many N- and Olinked glycans (Staudacher et al. 1999), and modifies serine and threonine residues in many EGF domaincontaining proteins (Harris and Spellman 1993). Fucose-containing glycans are implicated in developmental processes determined by the Notch family of signaling receptors (Bruckner et al. 2000; Chen et al. 2001; Moloney et al. 2000) and in Lewis X-dependent cell-cell interactions in the developing central nervous system (Ashwell and Mai 1997). Given the varied biological roles of fucosylated glycans during ontogeny, fucose deficiency is likely to present FX mutant mice with multiple hurdles to survival at numerous developmental stages.

What is the identity of the genetic loci controlling survival of FX mutant mice? Modifier genes could affect supply or utilization of cellular fucose, thereby altering the global level of fucosylation in $\mathrm{FX}+/-$ and FX-/- embryos and potentially affecting multiple fucose-regulated pathways. Alternatively, modifier genes could regulate critical developmental events by modulating specific downstream functions of fucosylated glycans without impact on embryonic or postnatal fucosylation. Thus, a considerable proportion of genes in the murine genome could be considered candidate modifier loci for control of FXmediated lethality.

Modifier effects are well described in the model genetic system Drosophila melanogaster, including several determined by carbohydrate-related genes. For instance, a hypomorphic allele of sugar less, a gene required for the synthesis of UDP-glucuronic acid, suppresses the white eye phenotype (Benevolenskaya et al. 1998), and mutations in the fringe connection gene encoding a Golgi UDP-sugar transporter modify the phenotypes of Notch pathway mutants (Goto et al. 2001; Selva et al. 2001). Less is known regarding potential sugar-related modifier genes in mammals. The Fuca gene encoding lysosomal $\alpha$-fucosidase represents a tantalizing candidate locus for modification of $F X$ mediated lethality since $\alpha$-fucosidase-dependent catabolism of endocytosed glycoproteins (Johnson and Alhadeff 1991) may release fucose to support 
the salvage pathway (Jonas et al. 1990) and since there is allelic variation in the Fuca locus among inbred mouse strains that alters fucosidase activity (Johnson and Hong 1986). However, SSLP markers in the vicinity of the Fuca locus on murine Chr 4 segregate independently from FX-/- survival (D.J. Becker and J.B. Lowe, unpublished data), making it unlikely that genetic variation in fucosidase activity accounts for FX-dependent embryonic lethality. In this context, it is important to point out that almost nothing is known about the genetics or biochemistry of the salvage pathway genes in mice. It thus remains possible that genetic variation in the loci that control this pathway may contribute to the genetic variability in lethality in these mice. Future studies to uncover the relative importance of these and other potential mechanisms of lethality modification in FX mutant mice have the potential to shed light on numerous aspects of carbohydrate metabolism and function.

\section{Acknowledgments}

We thank Ying Plews for help with genomic DNA preparation, Suzanne Genik for genotyping with fluorescent markers, Dave Siemeniak and Rich Raymond for technical advice, and Dr. Tom Glaser for helpful comments. This work was supported in part by National Institutes of Health grant 1P01CA71932 to J.B. Lowe, D.J. Becker was supported by a Rackham Predoctoral Fellowship from the Rackham Graduate School of the University of Michigan. J.B. Lowe and D. Ginsburg are Investigators of the Howard Hughes Medical Institute.

\section{References}

1. Ashwell KW, Mai JK (1997) Developmental expression of the CD 15 epitope in the hippocampus of the mouse. Cell Tissue Res 289, 17-23

2. Benevolenskaya EV, Frolov MV, Birchler JA (1998) The sugarless mutation affects the expression of the white eye color gene in Drosophila melanogaster. Mol Gen Genet 260, 131-143

3. Bruckner K, Perez L, Clausen H, Cohen S (2000) Glycosyltransferase activity of Fringe modulates NotchDelta interactions. Nature 406, 411-415

4. Chen J, Moloney DJ, Stanley P (2001) Fringe modulation of Jagged1-induced Notch signaling requires the action of beta 4galactosyltransferase-1. Proc Natl Acad Sci USA 98, 13716-13721

5. Dipple KM, McCabe ER (2000a) Modifier genes convert "simple" Mendelian disorders to complex traits. Mol Genet Metab 71, 43-50

6. Dipple KM, McCabe ER (2000b) Phenotypes of patients with "simple" Mendelian disorders are complex traits: thresholds, modifiers, and systems dynamics. Am J Hum Genet 66, 1729-1735

7. Eshel R, Zanin A, Sagi-Assif O, Meshel T, Smorodinsky NI et al. (2000) The GPI-linked Ly-6 antigen E48 regulates expression levels of the FX enzyme and of Eselectin ligands on head and neck squamous carcinoma cells. J Biol Chem 275, 12833-12840

8. Goto S, Taniguchi M, Muraoka M, Toyoda H, Sado Y et al. (2001) UDP- sugar transporter implicated in glycosylation and processing of Notch. Nat Cell Biol 3, 816-822

9. Harris RJ, Spellman MW (1993) O-linked fucose and other post-translational modifications unique to EGF modules. Glycobiology 3, 219-224

10. Johnson SW, Alhadeff JA (1991) Mammalian alpha-Lfucosidases. Comp Biochem Physiol B 99, 479-488

11. Johnson WG, Hong JL (1986) Variation in alpha-L-fucosidase properties among 28 inbred mouse strains: six strains have high enzyme activity and heat-stabile enzyme with a variant pH-activity curve; twenty-two strains have low activity and heat-labile enzyme. Biochem Genet 24, 469-483

12. Jonas AJ, Conrad P, Jobe H (1990) Neutral-sugar transport by rat liver lysosomes. Biochem 272, 323-326

13. Kappas A, Sassa S, Galbraith RA, Nordmann Y (1995) The porphyrias. In: The Metabolic and Molecular $\mathrm{Ba}$ ses of Inherited Disease, CR Scriver, AL Beaudet, WS Sly, and D Valle, (eds.) (New York: McGraw-Hill, Inc), pp 2103-2159

14. Keppler OT, Hinderlich S, Langner J, Schwartz-Albiez R, Reutter W, et al. (1999) UDP-G1cNAc 2-epimerase: a regulator of cell surface sialylation. Science 284, 1372-1376

15. Marquardt T, Luhn K, Srikrishna G, Freeze HH, Harms E, et al. (1999) Correction of leukocyte adhesion deficiency type II with oral fucose. Blood 94, 3976-3985

16. Moloney DJ, Panin VM, Johnston SH, Chen J, Shao et al. (2000) Fringe is a glycosyltransferase that modifies Notch. Nature 406, 369-375

17. Nadeau JH (2001) Modifier genes in mice and humans. Nat Rev Genet 2, 165-174

18. Pearson H (2002) Surviving a knockout blow. Nature 415, 8-9

19. Puglielli L, Hirschberg CB (1999) Reconstitution, identification, and purification of the rat liver golgi membrane GDP-fucose transporter. J Biol Chem 274, 35596-35600

20. Scriver CR, Waters PJ (1999) Monogenic traits are not simple: lessons from phenylketonuria. Trends Genet $15,267-272$

21. Selva EM, Hong K, Baeg GH, Beverley SM, Turco SJ et al. (2001) Dual role of the fringe connection gene in both heparan sulphate and fringe-dependent signalling events. Nat Cell Biol 3, 809-815

22. Simpson EM, Linder CC, Sargent EE, Davisson MT, Mobraaten LE et al. (1997) Genetic variation among 129 substrains and its importance for targeted mutagenesis in mice. Nat Genet 16, 809-815

23. Smith PL, Myers JT, Rogers CE, Zhou L, Petryniak B et al. (2002) Conditional control of selectin ligand ex- 
pression and global fucosylation events in mice with a targeted mutation at the FX locus. J Cell Biol 158, 801-815

24. Staudacher E, Altmann F, Wilson IB, Marz L (1999) Fucose in N-glycans: from plant to man Biochim Biophys Acta 1473, 216-236

25. Strauss WM (1998) Preparation of genomic DNA from mammalian tissue. In Current Protocols in Molecular Biology. FM. Ausubel, R. Brent, RE, Kingston, DD Moore, JG Seidman et al., (eds.) (New York: John J. Wiley and Sons, Inc), pp 2.2.1-2.2.3
26. Taylor BA, Navin A, Phillips SJ (1994) PCR-amplification of simple sequence repeat variants from pooled DNA samples for rapidly mapping new mutations of the mouse. Genomics 21, 626-632

27. Toma L, Pinhal MA, Dietrich CP, Nader HB, Hirschberg CB (1996) Transport of UDP-galactose into the Golgi lumen regulates the biosynthesis of proteoglycans. J Biol Chem 271, 3897-3901

28. Tonetti M, Sturla L, Bisso A, Benatti U, De Flora A (1996) Synthesis of GDP-L-fucose by the human FX protein. J Biol Chem 271, 27274-27279 